Research article

\title{
Increased risk of traffic accidents in subjects with latent toxoplasmosis: a retrospective case-control study Jaroslav Flegr*1, Jan Havlícek ${ }^{2}$, Petr Kodym³ ${ }^{3}$, Marek Malý ${ }^{4}$ and Zbyněk Smahel 5
}

Address: ${ }^{1}$ Department of Parasitology, Faculty of Science, Charles University, Prague, Czech Republic, ${ }^{2}$ Research Centrum of Personality and Ethnic Studies, Faculty of Humanities, Charles University, Prague, Czech Republic, ${ }^{3}$ National Reference Laboratory for Toxoplasmosis, National Institute of Public Health, Prague, Czech Republic, ${ }^{4}$ Department of Biostatistics, National Institute of Public Health, Prague, Czech Republic and ${ }^{5}$ Department of Anthropology and Human Genetics, Faculty of Science, Charles University, Prague, Czech Republic

E-mail: Jaroslav Flegr* - flegr@cesnet.cz; Jan Havlícek - havel@natur.cuni.cz; Petr Kodym - kodym@szu.cz; Marek Malý - mmaly@szu.cz; Zbyněk Smahel - smahel@natur.cuni.cz

*Corresponding author

Published: 2 July 2002

Received: 19 April 2002

BMC Infectious Diseases 2002, 2:11

Accepted: 2 July 2002

This article is available from: http://www.biomedcentral.com/I47|-2334/2/I I

(C) 2002 Flegr et al; licensee BioMed Central Ltd. Verbatim copying and redistribution of this article are permitted in any medium for any purpose, provided this notice is preserved along with the article's original URL.

\begin{abstract}
Background: The parasite Toxoplasma gondii infects 30-60\% of humans worldwide. Latent toxoplasmosis, i.e., the life-long presence of Toxoplasma cysts in neural and muscular tissues, leads to prolongation of reaction times in infected subjects. It is not known, however, whether the changes observed in the laboratory influence the performance of subjects in real-life situations.
\end{abstract}

Methods: The seroprevalence of latent toxoplasmosis in subjects involved in traffic accidents ( $\mathrm{N}$ $=146)$ and in the general population living in the same area $(N=446)$ was compared by a MantelHaenszel test for age-stratified data. Correlation between relative risk of traffic accidents and level of anti-Toxoplasma antibody titre was evaluated with the Cochran-Armitage test for trends.

Results: A higher seroprevalence was found in the traffic accident set than in the general population $\left(\mathrm{Chi}^{2} \mathrm{MH}=2 \mathrm{I} .45, \mathrm{p}<0.000 \mathrm{I}\right)$. The value of the odds ratio $(\mathrm{OR})$ suggests that subjects with latent toxoplasmosis had a $2.65\left(\mathrm{C} . \mathrm{I}_{.95}=\mathrm{I} .76-4.0 \mathrm{I}\right)$ times higher risk of an accident than the toxoplasmosis-negative subjects. The $\mathrm{OR}$ significantly increased with level of anti-Toxoplasma antibody titre $(p<0.000 \mathrm{I})$, being low $(\mathrm{OR}=1.86, \mathrm{C} . \mathrm{I} .95=\mathrm{I} . \mathrm{I} 4-3.03)$ for the 99 subjects with low antibody titres ( 8 and 16$)$, higher $(\mathrm{OR}=4.78$, C.I.95 $=2.39-9.59)$ for the 37 subjects with moderate titres $(32$ and 64$)$, and very high $(O R=16.03$, C.I.95 $=1.89-135.66)$ for the 6 subjects with titres higher than 64.

Conclusion: The subjects with latent toxoplasmosis have significantly increased risk of traffic accidents than the noninfected subjects. Relative risk of traffic accidents decreases with the duration of infection. These results suggest that 'asymptomatic' acquired toxoplasmosis might in fact represent a serious and highly underestimated public health problem, as well as an economic problem. 


\section{Background}

The parasitic protozoon Toxoplasma gondii (phylum Apicomplexa) infects various warm-blooded vertebrates, including 30-60\% of humans in most countries [1]. After a short phase of acute toxoplasmosis the infection proceeds into its latent phase when cysts are formed and these survive for the rest of the host's life, mainly in neural and muscular tissues. In immunocompetent human subjects the latent phase of infection is usually considered asymptomatic and harmless [2].

Toxoplasma is known to induce specific behavioural changes in infected rodents. Observed decreases in motor performance [3], learning capacity [4], neophobia [5], and fear [6], and increases in activity $[7,8]$, and reaction times [9] are usually considered evolutionary adaptations of the parasite that facilitate the transmission from intermediate to definitive host (typically from rodents to cat) by predation [10]. Human latent toxoplasmosis leads to prolongation of reaction times [11] and changes in personality profiles $[12,13]$. These changes are probably side effects either of the rodent-aimed manipulative activity of Toxoplasma or of some pathogenic activity of the parasites in the brain. The changes cannot influence the risk of predation in modern humans; nevertheless, prolongation of reaction times could increase the risk of other incidents such as traffic accidents. If this is true then the prevalence of toxoplasmosis in participants in traffic accidents should be higher than in the general population living in the same area.

Here we report the results of retrospective case-control study that compares the seroprevalence of toxoplasmosis in persons injured in traffic accidents with the seroprevalence of toxoplasmosis in general population living in the same area.

\section{Methods \\ Study population}

A) Traffic accident sample

This group consisted of 85 men and 61 women, outpatients at the Surgery Unit of Královské Vinohrady Hospital, one of two principal hospitals serving central Prague. Only residents of central Prague districts, 15-70 years old, with Czech citizenship and with a negative result of the laboratory test for alcohol (only 28 subjects were tested for alcohol) were included in the study. We included only the persons who could have actively influenced the probability of their accidents. All pedestrians hit by cars or trams on a carriage-way were included because, according to the Czech laws on road traffic valid until January 2001, pedestrians were obliged to give way to all approaching vehicles before entering the carriage-way, even on the pedestrian crossing. Drivers whose car was hit from the side or back or whose records contained the information that they did not cause the accident (the information in records was judged by three independent persons) were excluded from the sample before the results of their tests for toxoplasmosis were known. The series included all patients meeting the criteria listed including pedestrians and drivers who were involved in the same experiment. The serological testing for toxoplasmosis was performed using part of the blood samples collected for other purposes (e.g., testing for alcohol) between 1997-2000.

\section{B) Controls}

The controls consisted of 230 men and 216 women, residents of central Prague districts, selected by quota sampling and screened for toxoplasmosis in the 1990 and 1996 multipurpose immunological surveys (MIS) [14]. Samples of sera were collected by district and/or paediatric practitioners from about 20 randomly selected medical districts in the studied regions. Each participating medical practitioner was instructed to obtain quotas according to age group and gender from subjects without acute clinical symptoms.

\section{Serological tests}

All sera sent to the National Reference Laboratory for Toxoplasmosis in the National Institute of Public Health were examined by complement-fixation test (CFT) using a commercial CFT kit (SEVAC, Prague), by IgG ELISA (SEVAC, Prague), and by IgM ELISA (TestLine, Brno). The positive result of a CFT; (titres 8 and higher), together with a negative IgM ELISA test (positivity index<0.9), was considered here as indicative of latent toxoplasmosis. The CFT has been used as the principal screening method for toxoplasmosis in all serological surveys and basic-research projects in the Czech Republic since 1972, which makes it possible to compare the results of different studies and to study long-term trends. There is a very good agreement between qualitative results of CFT and IgG ELISA methods. The CFT titre 8 corresponds to concentrations of Toxoplasma-specific IgG of about $10 \mathrm{IU} / \mathrm{ml}$ as measured with IgG ELISA.

\section{Statistical analysis}

Because of a low probability of traffic accident, the odds ratio (OR) can be used as a very good approximation of the relative risk in this retrospective case-control study [15]. The Mantel-Haenszel inference for age-stratified data was used to test statistical significance of association between two binary variables, i.e., the risk factor (latent toxoplasmosis) and the response (traffic accident), in the 2by- 2 contingency table and for estimation of the corresponding common OR. For the analysis of particular age strata, the non-stratified version of OR was used. The homogeneity of ORs in different age strata, in drivers/pedestrians, and in male/female strata was estimated on the basis of Zelen exact tests. The hypothesis that the risk of 
Table I: Odds ratios for a traffic accident for Toxoplasma-infected subjects

\begin{tabular}{|c|c|c|c|c|c|}
\hline Subset & $N$ victims & $N$ controls & Odds Ratio & C.I.(95\%) & $P$ \\
\hline $15-29$ years & $59[21,35.6 \%]$ & $249[38,15.3 \%]$ & 3.07 & $1.63-5.79$ & $<0.001$ \\
\hline 30-44 years & $25[9,36.0 \%]$ & $67[15,22.4 \%]$ & 1.95 & $0.72-5.29$ & 0.190 \\
\hline 45-59 years & $39[17,43.6 \%]$ & $90[22,24.4 \%]$ & 2.39 & $1.08-5.29$ & 0.032 \\
\hline $60-70$ years & $23[11,47.8 \%]$ & $40[9,22.5 \%]$ & 3.16 & $1.05-9.53$ & 0.041 \\
\hline Total & $146[58,39.7 \%]$ & $446[84,18.8 \%]$ & 2.65 & $1.76-4.01$ & $<0.001$ \\
\hline Men & $85[30,35.3 \%]$ & $230[39,16.9 \%]$ & 2.71 & $1.54-4.79$ & $<0.001$ \\
\hline Women & $6 \mathrm{I}[28,45.9 \%]$ & $216[45,20.8 \%]$ & 2.68 & $1.43-5.02$ & 0.002 \\
\hline Drivers & $90[35,38.9 \%]$ & $446^{*}[84,18.8 \%]$ & 2.75 & $1.68-4.48$ & $<0.001$ \\
\hline Pedestrians & $56[23,4 \mid .1 \%]$ & $446^{*}[84,18.8 \%]$ & 2.57 & $1.39-4.78$ & 0.003 \\
\hline
\end{tabular}

\footnotetext{
* We used all 446 subjects as controls in the marked tests, as no information was available on the driver/pedestrian status of subjects in the control set. It seems reasonable to suppose that prevalence of latent toxoplasmosis is similar in pedestrians and drivers in the control set. Odds ratios (ORs), i.e., the odds in favour of traffic accident for the Toxoplasma-infected subjects divided by the odds in favour of traffic accident for the Toxoplasma-negative subjects, are listed for particular age strata; Mantel-Haenszel inferences of a common OR estimate (taking into account age-stratification of the data) are listed for other categories of subjects. The numbers in brackets in columns 2 and 3 show the number (and percentage) of Toxoplasma-positive subjects. All p-values are given for two-tailed tests.
}

traffic accident increases with the level of anti-Toxoplasma antibody titre was evaluated with the exact Cochran-Armitage test for trend and the hypothesis of increased average age in the accident group in comparison with controls within particular age strata was tested using a one-tailed age-stratified permutation test with raw observations (age) themselves as the scores (Pitman's test), i.e. exact alternative to stratified t-test.

All statistical testing was done with StatXact ${ }^{\circledR}$ 4.0.1. Whenever possible, the exact tests as well as the asymptotic tests were performed; the results of exact and asymptotic tests were usually very similar.

\section{Results}

The seroprevalence of latent toxoplasmosis in subjects involved in traffic accidents and in the general population was compared by a Mantel-Haenszel test for age-stratified data (Table 1, Fig. 1). The result suggests a significantly higher seroprevalence in the traffic accident set $\left(\mathrm{Chi}^{2} \mathrm{MH}=\right.$ $21.45, \mathrm{p}<0.0001)$. The value of the odds ratio (OR) suggests that subjects with latent toxoplasmosis had a 2.65 (C.I. $\left.{ }^{9}=1.76-4.01\right)$ times higher risk of a traffic accident than the toxoplasmosis-negative subjects. The ORs varied between particular age strata; however, the test of homogeneity of odds ratios shows that these differences are not significant (Zelen statistics $=0.0076, \mathrm{p}=0.88$ ). Analyses of the traffic accident sample found no significant differences in ORs between drivers and pedestrians or between men and women. To check the robustness of our results, we repeated all analyses with six different age strata (15$19,20-29,30-39,40-49,50-59$, and $60-70$ years). The results were very similar to those reported in Table 1 .
However, due to the low number of observations in some strata, the relative risks in particular age strata fluctuated. (The total $\mathrm{OR}=2.48$, C.I. $95=1.64-3.77 ;$ age $15-19, \mathrm{OR}=$ 1.87, C.I.95 $=0.36-9.73$; age 20-29, OR = 2.79, C.I.95 = 1.35-5.78; age 30-39, OR = 1.06, C.I.95 = 0.28-3.96; age $40-49, \mathrm{OR}=4.04$, C.I. $95=1.32-12.39 ;$ age $50-59, \mathrm{OR}=$ 2.12, C.I. $.95=0.83-5.43$; and age 60-70, OR = 3.16, C.I. 95 $=1.05-9.52$.) Again, there was no significant difference in age of the accident and control groups within these age strata $(\mathrm{p}=0.25$, Pitman's exact test $)$.

Positive correlation between the subjects' age and the probability of being latent toxoplasmosis-positive in the Czech republic was reported [14] and some correlation between the probability of traffic accident and the subjects' age could also exist. Therefore, increased risk of traffic accident of subject with latent toxoplasmosis can be theoretically caused by higher average age of traffic accident victims than of the controls within particular agestrata. To test this hypothesis, we checked the data for increased age of traffic accident victims in comparison with controls within particular age strata using a one-tailed Pitman's test. The results suggest that the age of the accident group was only slightly higher than the age of controls ( $\mathrm{p}$ $=0.15$, exact test). The Pitman's tests for particular age strata show that only for the first stratum (15-29 years) was the mean age of the accident group (22.7 years) significantly higher than the average age of controls $(20.2$ years, ( $p=0.028$, Pitman's exact test). Therefore, this factor cannot be responsible for the higher prevalence of latent toxoplasmosis in the accident group in three other strata. 


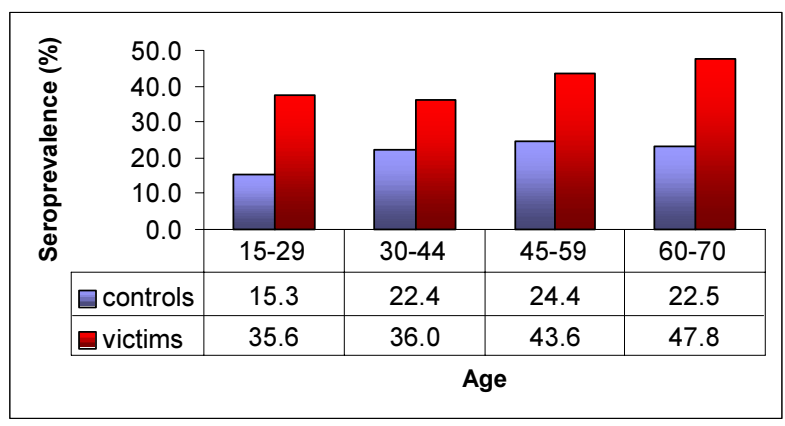

\section{Figure I}

Seroprevalence of latent toxoplasmosis The figure shows difference in seroprevalence of latent toxoplasmosis (\%) between the representative sample of general Prague residents (controls) and the sample of Prague residents involved in traffic accidents between 1997-2000 (victims). For numbers of subjects in particular categories, see Table I.

The OR significantly increased with level of anti-Toxoplas$m a$ antibody titre $(\mathrm{p}<0.0001$, exact Cochran-Armitage trend test; Fig. 2), being low ( $\mathrm{OR}=1.86$, C.I.95 $=1.14-$ 3.03 ) for the 99 subjects with low antibody titres ( 8 and $16)$, higher $(\mathrm{OR}=4.78$, C.I. $95=2.39-9.59)$ for the 37 subjects with moderate titres (32 and 64), and very high (OR $=16.03$, C.I.95 $=1.89-135.66)$ for the 6 subjects with titres higher than 64 . In fact, of these six subjects with high titres, i.e., subjects with supposedly relatively recent or massive T. gondii infection, five were found among the 146 traffic accident participants and only one among the 446 controls.

\section{Discussion}

The increased seroprevalence of toxoplasmosis in victims of traffic accidents suggested that the subjects with latent toxoplasmosis had significantly higher risk of traffic accidents than non-infected subjects. Relative risk of traffic accidents was highest in subjects with supposedly relatively recent or massive $T$. gondii infection and decreased with the decrease of anti-toxoplasma immunity, i.e. with duration of Toxoplasma infection.

Retrospective case-control study cannot reveal why a higher seroprevalence of latent toxoplasmosis occurs among victims of traffic accidents. Theoretically, toxoplasmosis can either increase the probability of traffic accident or decrease the probability that the subject visits a physician and therefore enters the control set. Results of independent recent studies on prevalence of toxoplasmosis in Prague, however, suggest that the former possibility is more probable. After taking into account the differences in age and gender composition of particular sets, the differences between seroprevalence measured in the multi- purpose immunological surveys and in the set of 857 conscripts or in the set of 723 pregnant women were not significant (conscripts: $p=0.12$, women: $p=0.78$ ) [16]. It is also possible that some unknown confounding factor, such as the socioeconomic status of a person, can influence the probability of both Toxoplasma infection and a traffic accident. The most parsimonious explanation, however, seems to be that the Toxoplasma-infected individuals have increased risk of traffic accidents due to their worse psychomotor performance. Previously published results suggest that the effect of latent toxoplasmosis on human reaction times is rather moderate, accounting for only $8 \%$ of the total variance [11]. However, the comparison of psychomotor performance in the first, second, and third minutes of the test indicates that Toxoplasma-infected subjects have reasonably good reaction times in the first minute of the test but lose concentration more quickly than the controls. It has been reported in literature that older drivers are able to compensate for the prolongation of their reaction times [17]. It suggests that other behavioural factors, like the capacity for long-term concentration, are more critical with respect to the risk of a traffic accident [19]. It is difficult to quantify capacity for longterm concentration under natural conditions using laboratory test. However, it is possible that the risk of traffic accident is in fact more strongly influenced by the toxoplasmosis-associated decrease of capacity of concentration than by the moderate increase of reaction time.

The existence of positive correlation between concentration of antibodies and relative risk of traffic accident contrasts with previously reported negative correlation between concentration of antibodies and increase of reaction times of infected subjects [11]. Possibly, the positive correlation between the concentration of antibody and the risk of traffic accident observed in the present study reflects a positive correlation between intensity of infection and its behavioural effect rather than a negative correlation between duration of infection and its behavioural effects. It is also possible that the long-term infected subjects with low antibody concentration already successfully reduced the risk of accident by adjusting their behaviour to the decrease of their psychomotor performance. In both studies, we eliminated all subjects with acute toxoplasmosis from the data set by screening the subjects for IgM antibodies. Moreover, in the present study, a positive correlation existed even within the subset of subjects with low titres $(8-32)(p=0.032)$. Therefore, the effect of latent toxoplasmosis, rather than transient effect of acute toxoplasmosis disease seems to be more probable candidate for explanation of increased risk of traffic accident in Toxoplasma-positive subjects.

The dormant stages of Toxoplasma, the tissue cysts, are located mainly in brain and muscular tissues of infected an- 


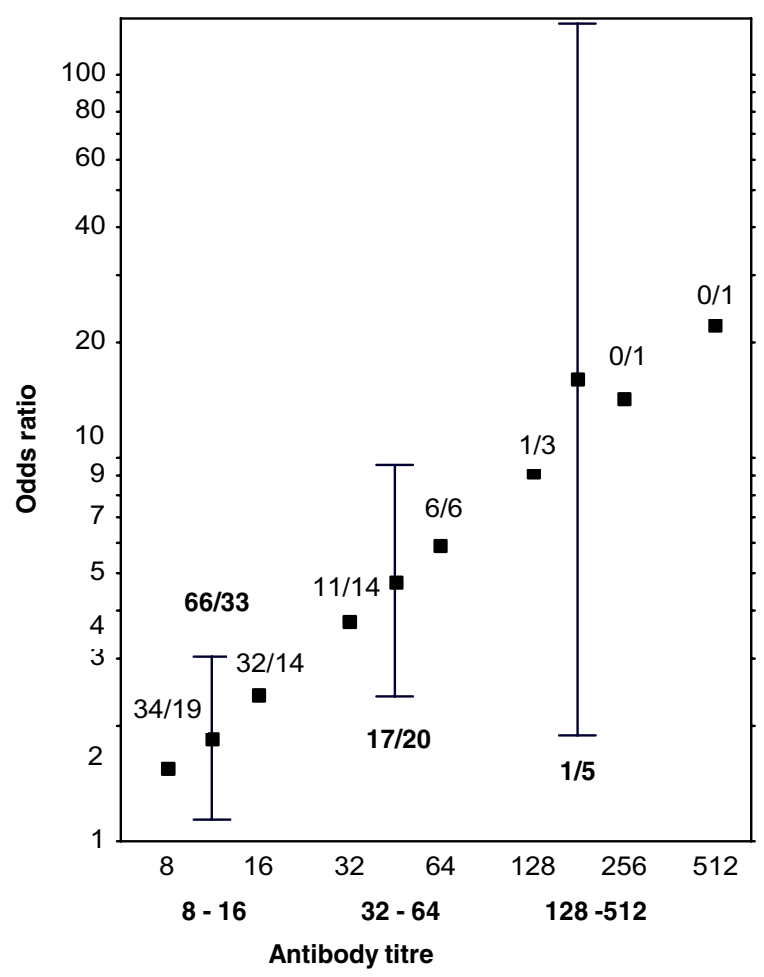

Figure 2

Positive concordance of ORs, reflecting relative risk of traffic accident, with anti-Toxoplasma antibody titre The figure shows the exact estimation of the ORs with their $95 \%$ confidence intervals. The numbers indicate number of subjects with particular titre (NSPT) among 446 controls (numerator) and NSPT among 146 victims (denominator). Statistical significance of the two-tailed exact CochranArmitage test for trend with age-stratified data was $P<$ 0.0001 .

imals [18]. In rodents, these cysts usually survive in an infectious form until the death of the host. The low rate of decrease of specific antibodies in an infected individual [19], extremely low frequency of seroconversion (loss of specific antibodies) among seropositive subjects [14], and high frequency of reactivation of toxoplasmosis in seropositive AIDS patients $[20,21]$ suggest the existence of lifelong Toxoplasma infections in humans as well. The slowing of reaction times and other behavioural changes that can increase the risk of predation in Toxoplasma-infected rodents are probably mediated by production or induction of production of a neurotransmitter, possibly dopamine, [22] in the brain of infected animals. In humans, such manipulative activity is non-productive for Toxoplasma. It must be considered here "an evolutionary constraint," as it arises as a side effect of selection in different host species, with no apparent direct adaptive significance in humans [23]. Indeed, the difference in behavioural alterations induced by Toxoplasma in various animal species might help to reveal the neurophysiological mechanism(s) of the manipulation activity of the parasite.

\section{Conclusions}

The subjects with latent toxoplasmosis have significantly increased risk of traffic accidents than the non-infected subjects. Because of its high prevalence and therefore extremely high attributable mortality, latent toxoplasmosis, the mildest form of $T$. gondii infection, might in fact represent a serious and highly underestimated economic and public health problem.

\section{Competing interests}

None declared.

\section{Authors' contributions}

JF designated the study, wrote the manuscript and participated in analyses. JH, PK and Z ̌S collected the data, participated in analyses and in manuscript writing. MM did statistical analyses.

All authors read and approved the final manuscript.

\section{Acknowledgements}

We thank D. Frynta and K. Zvára for their advice. This work was supported by the Czech Ministry of Education grant II 3 I-4.

\section{References}

I. Holliman RE: Toxoplasmosis, behaviour and personality. J Infect 1997, 35: 105-110

2. Remington JS: Toxoplasmosis in the adult. Bull New York Acad Med 1974, 50:21I-227

3. Hutchison WM, Aitken PP, Wells BWP: Chronic Toxoplasma infections and motor performance in the mouse. Ann Trop Med Parasitol 1980, 74:505-510

4. Witting P-A: Learning capacity and memory of normal and Toxoplasma-infected laboratory rats and mice. Zeitschr Parasitenkunde 1979, 6 I:29-5 |

5. Webster JP, Brunton CFA, Macdonald DW: Effect of Toxoplasmo gondii upon neophobic behaviour in wild brown rats, Rattus norvegicus. Parasitology 1994, 109:37-43

6. Berdoy M, Webster JP, Macdonald DW: Fatal attraction in rats infected with Toxoplasma gondii. Proc R Soc Lond B Biol Sci 2000, 267:1591-1594

7. Hay J, Hutchison WM, Aitken PP, Graham PP: The effect of congenital and adult-acquired Toxoplasma infections on activity and responsiveness to novel stimulation in mice. Ann Trop Med Parasitol 1 983, 77:483-495

8. Webster JP: The effect of Toxoplasma gondii and other parasites on activity levels in wild and hybrid Rattus norvegicus. Parasitology 1994, 109:583-589

9. Hrdá Š, Votýpka J, Kodym P, Flegr J: Transient nature of Toxoplasma gondii-induced behavioral changes in mice. J Parasitol 2000, 86:657-663

10. Barnard C], Behnke JM: Parasitism and host behavior. New York, Taylor and Francis 1990

II. Havliček J, Gašová Z, Smith AP, Zvára K, Flegr J: Decrease of psychomotor performance in subjects with latent "asymptomatic" toxoplasmosis. Parasitology 200 I, I 22:5I5-520

12. Flegr J, Zitková S, Kodym P, Frynta D: Induction of changes in human behaviour by the parasitic protozoan Toxoplasma gondii. Parasitology 1996, I I 3:49-54

13. Flegr J, Kodym P, Tolarová V: Correlation of duration of latent Toxoplasma gondii infection with personality changes in women. Biol Psychology 2000, 53:57-68 
14. Kodym P, Malý M, Švandová E, Ležatková $H$, Bažoutová M, Vlčková J, Beneš C, Zástěra M: Toxoplasma in the Czech Republic 19231999: First case to widespread outbreak. Int J Parasitol 200I, 31:125-132

15. Fisher LD, Van Belle G: Biostatistics. A methodology for the health sciences. New York, John Wiley \& Sons, Inc. 1993, 193-194

16. Flegr J, Havliček J: Changes in personality profile of young women with latent toxoplasmosis. Folia Parasitol 1999, 46:22-28

17. Petridou E, Moustaki M: Human factors in the causation of road traffic crashes. Europ J Epidemiol 2000, 16:819-826

18. Dubey JP: Tissue cyst tropism in Toxoplasma gondii : a comparison of tissue cyst formation in organs of cats, and rodents fed oocysts. Parasitology 1997, I I 5: 15-20

19. Konishi $\mathrm{E}$ : Annual change in immunoglobulin $\mathbf{G}$ and $\mathbf{M}$ antibody levels to Toxoplasma gondii in human sera. Microbiol Immunol 1989, 33:403-4II

20. Grant IH, Gold JW, Rosenblum M, Niedzwiecki D, Armstrong D: Toxoplasma gondii serology in HIV-infected patients: the development of central nervous system toxoplasmosis in AIDS. AIDS 1990, 4:519-521

21. Garly ML, Petersen E, Pedersen C, Lundgren JD, Gerstoft J: Toxoplasmosis in Danish AIDS patients. Scand J Infec Dis 1997, 29:597-600

22. Stibbs $\mathrm{HH}$ : Changes in brain concentrations of catecholamines and indoleamines in Toxoplasma gondii infected mice. Ann Trop Med Parasitol 1985, 79:153-157

23. Moore J, Gotteli NJ: A phylogenetic perspective on the evolution of altered host behaviours: a critical look at the manipulation hypothesis. In: Parasitism and Host Behaviour. (Edited by: Barnard CJ, Behnke JM) London: Taylor and Francis 1990, 193-230

\section{Pre-publication history}

The pre-publication history for this paper can be accessed here:

http://www.biomedcentral.com/1471-2334/2/11/prepub

Publish with BioMed Central and every scientist can read your work free of charge

"BioMedcentral will be the most significant development for disseminating the results of biomedical research in our lifetime."

Paul Nurse, Director-General, Imperial Cancer Research Fund

Publish with BMC and your research papers will be:

- available free of charge to the entire biomedical community

- peer reviewed and published immediately upon acceptance

- cited in PubMed and archived on PubMed Central

- yours - you keep the copyright 